\title{
Anatomía de la madera de cinco especies de Quintana Roo, México
}

\author{
Silvia Rebollar, CARMEN de la PAZ-PÉREZ Olvera y \\ ALEJANDRA QUINTANAR ${ }^{1}$
}

\begin{abstract}
RESUMEN. Se presenta la descripción anatómica macroscópica y microscópica de la madera de 5 especies procedentes de Puerto Morelos, Quintana Roo: Drypetes lateriflora (Euphorbiaceae), Exothea diphylla (Sapindaceae), Myrcianthes fragrans var. fragrans (Myrtaceae), Diospyros nicaraguensis (Ebenaceae) y Thevetia gaumeri (Apocynaceae). De cada especie se recolectó un árbol del cual se obtuvieron muestras de $15 \times 7 \times 1 \mathrm{~cm}$ con las que se hizo el estudio macroscópico y cubos de $2 \times 2 \mathrm{~cm}$ de los que se obtuvieron preparaciones fijas de cortes y material disociado para el estudio microscópico. A los elementos mensurables se les hizo un análisis estadístico y se consideró el valor de la media para su clasificación el cual se incluye junto con los valores mínimo y máximo de cađa descripción. Se proporciona un mapa del lugar de recolección, un cuadro y fotografías de la estructura microscópica de las especies.
\end{abstract}

ABSTRACT. Wood macroscopic and microscopic features of 5 species from Quintana Roo are given: Drypetes lateriflora (Euphorbiaceae), Exothea diphylla (Sapindaceae), Myrcianthes fragrans var. fragrans (Myrtaceae), Diospyros nicaraguensis (Ebenaceae) and Thevetia gaumeri (Apocynaceae). One tree from each species was colected; for the macroscopic study, wood samples of $15 \times 7 \times 1 \mathrm{~cm}$ of size were obtained and for the microscopic one wood samples of $2 \mathrm{X} 2 \mathrm{~cm}$ from which permanent slides and slash material were prepared. Measurable elements were submitted to a statistical anaylisis considering the media value for the classification including minima and maxima values in the description of each specie. A map pointing the collection place, a square and photographs of microscopic features of the species are given.

La estructura anatómica de la madera refleja características y propiedades cuyo conocimiento repercute en su aprovechamiento adecuado por lo que es importante realizar este tipo de estudios fundamentales en las especies cuya madera no es conocida.

${ }^{1}$ Departamento de Biología. División de C.B.S. Universidad Autónoma Metropolitana-Iztapalapa. Apdo. Postal 55-535; 09340, México, D.F. 
Los estudios realizados en especies de la Península de Yucatán abarcan aspectos anatómicos, tecnológicos o ambos. De especies recolectadas en Campeche están los de: Guridi (1968), Schultz y von Grotthus $(1968,1968$ a. y 1969), Torres (1969), Cárdenas (1971), Flores (1981) y Huerta y Becerra (1982); de Campeche y Quintana Roo los de: De La Paz-Pérez Olvera et al. $(1979,1980)$, Rogel (1982) y Corral (1985) y para Yucatán el de Rebollar et al. (1987).

En el estado de Quintana Roo la selva mediana subperennifolia está bien representada, con estratos arbóreos de 25 a 35 m (Miranda, 1978), con especies maderables sobreexplotadas y otras que aún no se aprovechan, aunque representen un potencial forestal y que son desperdiciadas al hacerse la tala selectiva (Halffter, 1980; Trueba, 1983). El desconocimiento de las características anatómicas de estas especies influye entre otros factores a su falta de comercialización; por esto se seleccionaron cinco especies que son abundantes, de amplia distribución y son usadas localmente (Escalante, 1986), para estudiar sus características macroscópicas y microscópicas.

\section{Metodología}

La madera de las especies estudiadas provino de 5 árboles sanos y de fustes rectos, uno para cada especie, procedentes de una selva mediana subperennifolia (Sánchez, 1987), que corresponde al Jardín Botánico Dr. Alfredo Barrera Marín, del Centro de Investigaciones de Quintana Roo, localizado en Puerto Morelos, Municipio Benito Juárez (fig. 1). La recolección fué hecha de acuerdo con las especificaciones propuestas por Ramos y Díaz (1981); las especies fueron identificadas y registradas tanto en la Xiloteca como en el Herbario Metropolitano de la Universidad Autónoma Metropolitana Iztapalapa.

De cada fuste se obtuvieron tablillas de xiloteca de $15 \times 7 \times 1 \mathrm{~cm}$, con las que se describieron las características macroscópicas; del mismo fuste se cortaron dos rodajas de $2 \mathrm{~cm}$ de grosor cada una donde se tomaron al azar cubos de $2 \times 2 \mathrm{~cm}$, para la obtención de cortes típicos de la madera y material disociado con los que se hizo el estudio de las características microscópicas, las cuales se ilustran con las fotografías correspondientes.

Los cubos se sometieron a un proceso de ablandamiento de acuerdo con Kukachka (1977), el cual varió según la especie; los cortes se hicieron en un microtomo de deslizamiento, se tiñeron con verde iodo, se deshidrataron y montaron con resina sintética. El material disociado se obtuvo por el método de Jeffrey (Johansen, 1940), se tiñó con pardo de Bismarck y se montó con gelatina glicerinada.

La denominación de las características macroscópicas se hizo siguiendo las tablas de Tortorelli (1956) y para el color se usaron las cartas de Munsell (1954). La visibilidad de los elementos se determinó en las caras transversales.

La nomenclatura utilizada en las descripciones microscópicas fué la de IAWA Committee (1989) y para los rayos se usó también la de Kribs (1968). La longitud de los 
ANATOMIA DE LA MADERA DE QUINTANA ROO

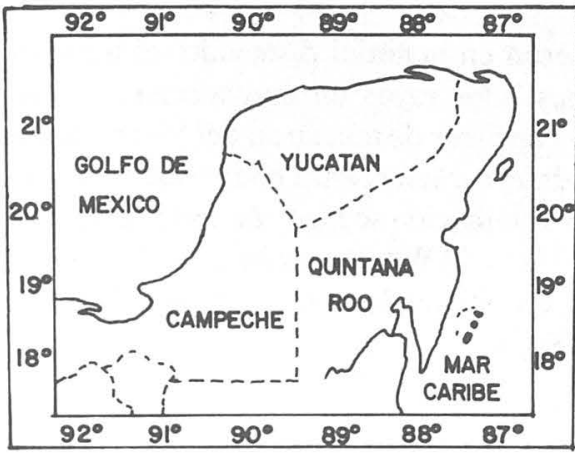

CANCUN

PUERTO

T

MORELOS
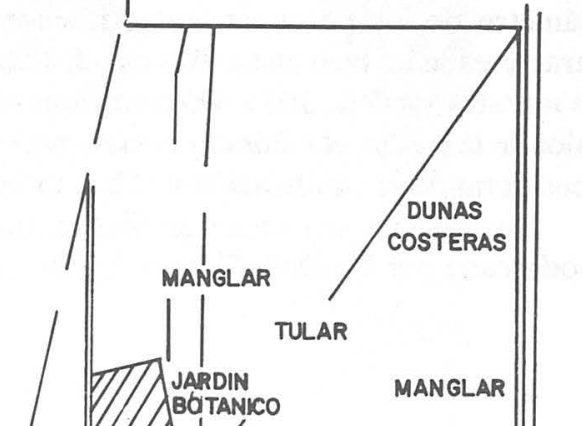

DUNAS ANGLAR COSTERAS

TULAR

MANGLAR

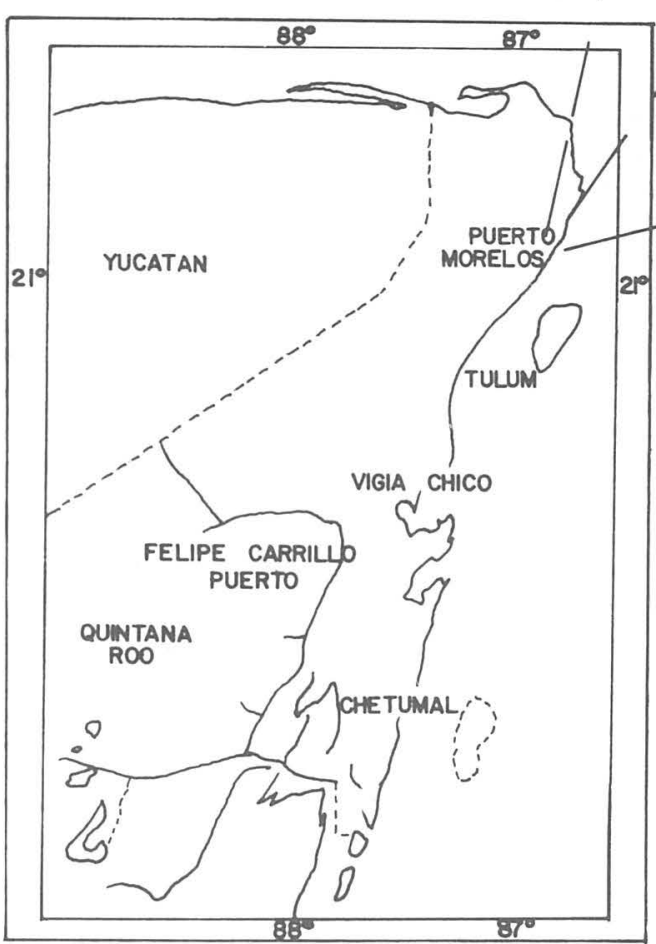

Fig. 1. Ubicación geográfica del área de recolección. 
vasos y las dimensiones de las fibras se midieron en material disociado; el número y diámetro de los poros en cortes transversales y los rayos en tangenciales. A estos caracteres se les hizo un análisis estadístico con un error de muestreo del 5\%; el tamaño de muestra varió de 100 a 400 mediciones según el carácter. Para cada especie se da el valor de la media, el mínimo y el máximo; su denominación se hizo con base en la media considerando la clasificación de Chattaway $(1932)$ y IAWA Committee $(1937,1939)$.

Las especies se ordenaron filogenéticamente siguiendo la clasificación de Engler modificada por De Dalla Torre y Harms (1963).

\section{RESUlTADOS}

\section{Drypetes lateriflora (Swartz) Krug. \& Urb. Euphorbiaceae}

Nombres comunes. Ekulub, sin che', xi'in che' (Campeche, Yucatán y Quintana Roo) (Sosa et al., 1985). 1949).

Distribución. Campeche, Yucatán, Quintana Roo y Chiapas (Standley y Steyermark,

\section{Características macroscópicas.}

La madera presenta albura y duramen bien delimitados; la albura mide $8 \mathrm{~cm}$, es de color castaño muy pálido (10YR 8/3) y gris claro (10YR 7/2), el duramen mide $7 \mathrm{~cm}$ y tiene varias tonalidades: castaño muy pálido (10YR 7/4), castaño (10YR 4/2), amarillo (2.5Y 8/8) y castaño grisáceo muy oscuro (10YR 3/2). El olor y el sabor no son característicos, el brillo es mediano, el veteado es pronunciado, la textura es fina y el hilo es recto.

Los poros, el parénquima axial, los rayos y las fibras son visibles con lupa; las zonas de crecimiento a simple vista.

Características microscópicas. (Lám. 1. figs. 1. a-c y cuadro 1).

La madera presenta porosidad difusa, la mayoría de los poros son múltiples radiales de 2 a 9 y pocos solitarios, muy numerosos $41(22-64) / \mathrm{mm} 2$, de diámetro tangencial muy pequeño 41 (15-61) $\mu \mathrm{m}$. Los elementos de vaso son de longitud mediana 725 (420-1400) $\mu \mathrm{m}$, sus paredes presentan puntuaciones areoladas alternas y su placa perforada es simple y escaleriforme de 2 a 6 barras, algunos elementos tienen una doble perforación en el mismo extremo. Presentan gomas y sustancias granulosas amarillas.

El parénquima axial es reticulado. Presenta cristales romboidales y sustancias granulosas amarillas.

Los rayos son de 1 a 3 series, la mayoría de 2, muy numerosos 18 (14-24)/mm, de tipo heterogéneo I y II; el cuerpo está formado por células procumbentes y los extremos por células verticales. Los uniseriados son extremadamente bajos 458 (140-1100) $\mu \mathrm{m}$, de 6 células de altura (1-25). Los poliseriados son muy bajos 632 (180-1450) $\mu \mathrm{m}$ y de anchura moderadamente fina 27 (15-45) $\mu \mathrm{m}$. Los uniseriados y los extremos uniseriados de los 

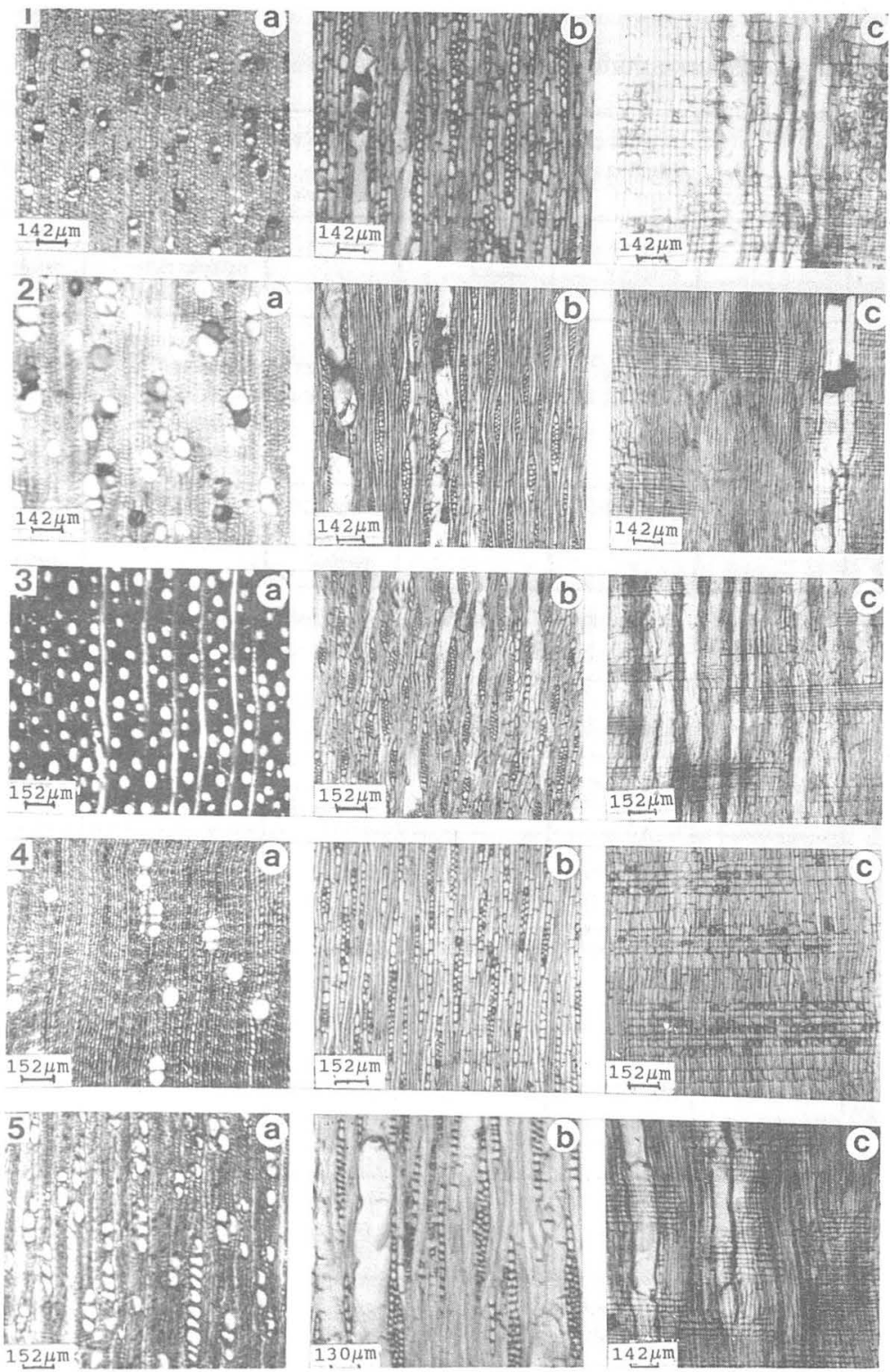

Lámina 1. figs. 1. a-c Drypetes lateriflora. figs. 2. a-c Exothea diphylla. figs. 3. a-c Myrcianthes fragrans var. fragrans. figs. 4. a-c Diospyros nicaraguensis. figs. 5. a-c Thevetia gaumeri. a. Corte transversal. b. Corte tangencial. c. Corte radial. 
CUADRO 1. Características microscópicas de las especies.

\begin{tabular}{|c|c|c|c|c|c|c|c|}
\hline & & & $\begin{array}{l}\text { Drypetes } \\
\text { lateriflora }\end{array}$ & $\begin{array}{l}\text { Exothea } \\
\text { diphylla }\end{array}$ & $\begin{array}{l}\text { Myrcianthes } \\
\text { fragans } \\
\text { var fragans }\end{array}$ & $\begin{array}{l}\text { Diospyros } \\
\text { nicaraguensis }\end{array}$ & $\begin{array}{l}\text { Thevetia } \\
\text { gaumeri }\end{array}$ \\
\hline \multirow{3}{*}{\multicolumn{2}{|c|}{$\begin{array}{l}\mathrm{P} \\
\mathrm{O} \\
\mathrm{R} \\
\mathrm{O} \\
\mathrm{S}\end{array}$}} & Agrupación & $\begin{array}{l}\text { múltiples } \\
\text { radiales y } \\
\text { solitarios }\end{array}$ & $\begin{array}{l}\text { solitarios y } \\
\text { múltiples } \\
\text { radiales }\end{array}$ & solitarios & $\begin{array}{l}\text { solitarios } \\
\text { múltiples radia- } \\
\text { les y agrupados }\end{array}$ & $\begin{array}{l}\text { múltiples } \\
\text { radiales solita- } \\
\text { rios y agrupados }\end{array}$ \\
\hline & & $\mathrm{N}^{\mathrm{0}} \times \mathrm{mm}^{2}$ & $\begin{array}{l}\text { muy } \\
\text { numerosos }\end{array}$ & numerosos & $\begin{array}{l}\text { muy } \\
\text { numerosos }\end{array}$ & $\begin{array}{l}\text { moderadamente } \\
\text { numerosos }\end{array}$ & $\begin{array}{l}\text { muy } \\
\text { numerosos }\end{array}$ \\
\hline & & Diámetro & $\begin{array}{l}\text { muy } \\
\text { pequeño }\end{array}$ & $\begin{array}{l}\text { moderadamen- } \\
\text { te pequeño }\end{array}$ & $\begin{array}{l}\text { muy } \\
\text { pequeño }\end{array}$ & $\begin{array}{l}\text { moderadamen- } \\
\text { te pequerio }\end{array}$ & $\begin{array}{l}\text { moderadamen- } \\
\text { te pequeño }\end{array}$ \\
\hline \multirow{5}{*}{$\begin{array}{l}\mathrm{P} \\
\mathrm{A} \\
\mathrm{R} \\
\mathrm{E} \\
\mathrm{N} \\
\mathrm{Q} \\
\mathrm{U} \\
\mathrm{I} \\
\mathrm{M} \\
\mathrm{A}\end{array}$} & $\begin{array}{l}\mathrm{A} \\
\mathrm{X} \\
\mathrm{1} \\
\mathrm{A} \\
\mathrm{L}\end{array}$ & Tipo & reticulado & unilateral & $\begin{array}{l}\text { unilateral, } \\
\text { reticulado y } \\
\text { marginal }\end{array}$ & $\begin{array}{l}\text { en bandas y } \\
\text { vasióntrico }\end{array}$ & $\begin{array}{l}\text { difuso } \\
\text { unilateral y } \\
\text { marginal }\end{array}$ \\
\hline & \multirow{4}{*}{$\begin{array}{l}\mathrm{R} \\
\mathrm{A} \\
\mathrm{D} \\
\mathrm{I} \\
\mathrm{A} \\
\mathrm{L}\end{array}$} & Tipo & $\begin{array}{l}\text { heterogéneos } \\
\text { Iy II }\end{array}$ & $\begin{array}{l}\text { heterogéneos } \\
\text { III }\end{array}$ & $\begin{array}{l}\text { heterogéneos } \\
\text { II y III }\end{array}$ & $\begin{array}{l}\text { heterogéneos } \\
\text { Iy III }\end{array}$ & $\begin{array}{l}\text { heterogéneos } \\
\text { II y III }\end{array}$ \\
\hline & & $\mathrm{N}^{\mathrm{x}} \mathrm{xmm}$ & $\begin{array}{l}\text { muy } \\
\text { numerosos }\end{array}$ & numerosos & numerosos & $\begin{array}{l}\text { muy } \\
\text { numerosos }\end{array}$ & numerosos \\
\hline & & Altura & muy bajos & $\begin{array}{l}\text { extremada- } \\
\text { mente bajos }\end{array}$ & $\begin{array}{l}\text { extremada- } \\
\text { mente bajos }\end{array}$ & muy bajos & $\begin{array}{l}\text { extremada- } \\
\text { mente bajos }\end{array}$ \\
\hline & & Anchura & $\begin{array}{l}\text { moderada- } \\
\text { mente finos }\end{array}$ & $\begin{array}{l}\text { muy } \\
\text { finos }\end{array}$ & $\begin{array}{l}\text { muy } \\
\text { finos }\end{array}$ & $\begin{array}{l}\text { moderada- } \\
\text { mente finos }\end{array}$ & $\begin{array}{l}\text { moderada- } \\
\text { mente finos }\end{array}$ \\
\hline \multirow{3}{*}{\multicolumn{2}{|c|}{$\begin{array}{l}\text { F } \\
\text { I } \\
\text { B } \\
\text { R } \\
\text { A } \\
\text { S }\end{array}$}} & Tipo & libriformes & $\begin{array}{l}\text { libriformes } \\
\text { septadas y fi- } \\
\text { brotaqueidas }\end{array}$ & libriformes & libriformes & libriformes \\
\hline & & Longitud & $\begin{array}{l}\text { moderada- } \\
\text { mente largas }\end{array}$ & $\begin{array}{l}\text { moderada- } \\
\text { mente cortas }\end{array}$ & $\begin{array}{l}\text { moderada- } \\
\text { mente largas }\end{array}$ & medianas & $\begin{array}{l}\text { moderada- } \\
\text { mente largas }\end{array}$ \\
\hline & & Pared & muy gruesa & delgada & muy gruesa & delgada & delgada \\
\hline \multirow{4}{*}{$\begin{array}{l}\mathrm{C} \\
\mathrm{O} \\
\mathrm{N} \\
\mathrm{T} \\
\mathrm{E} \\
\mathrm{N} \\
\mathrm{I} \\
\mathrm{D} \\
\mathrm{O} \\
\mathrm{S}\end{array}$} & & Gomas & en vasos & \begin{tabular}{l|} 
en vasos y \\
parénquima radial \\
radial
\end{tabular} & $\begin{array}{l}\text { en } \\
\text { parénquima } \\
\text { radial }\end{array}$ & $\begin{array}{l}\text { en vasos y } \\
\text { parénquima } \\
\text { radial }\end{array}$ & no presenta \\
\hline & & Cristales & $\begin{array}{l}\text { en parénquima } \\
\text { axial y radial }\end{array}$ & $\begin{array}{l}\text { no } \\
\text { presenta }\end{array}$ & $\begin{array}{l}\text { no } \\
\text { presenta }\end{array}$ & $\begin{array}{l}\text { en parénquima } \\
\text { axial y radial }\end{array}$ & $\begin{array}{l}\text { en parénquima } \\
\text { axial }\end{array}$ \\
\hline & & $\begin{array}{l}\text { Sustancias } \\
\text { granulosas } \\
\text { amarillas }\end{array}$ & $\begin{array}{l}\text { en vasos, parén- } \\
\text { quima axial, ra } \\
\text { dial y en fibras }\end{array}$ & $\begin{array}{l}\text { no } \\
\text { presenta }\end{array}$ & $\begin{array}{l}\text { no } \\
\text { presenta }\end{array}$ & $\begin{array}{l}\text { no } \\
\text { presenta }\end{array}$ & $\begin{array}{l}\text { no } \\
\text { presenta }\end{array}$ \\
\hline & & $\begin{array}{l}\text { Sustancias } \\
\text { Amarillas }\end{array}$ & $\begin{array}{l}\text { no } \\
\text { presenta }\end{array}$ & $\begin{array}{l}\text { no } \\
\text { presenta }\end{array}$ & $\begin{array}{l}\text { по } \\
\text { presenta }\end{array}$ & $\begin{array}{l}\text { no } \\
\text { presenta }\end{array}$ & $\begin{array}{l}\text { no } \\
\text { presenta }\end{array}$ \\
\hline
\end{tabular}


rayos poliseriados presentan abundantes cristales romboidales y sustancias granulosas amarillas.

Las fibras son de tipo libriforme, moderadamente largas 1457 (1100-1900) $\mu \mathrm{m}$, de diámetro fino 18 (13-25) $\mu \mathrm{m}$ y paredes muy gruesas 10 (5-13) $\mu \mathrm{m}$. Presentan sustancias granulosas amarillas.

Usos locales.

Los dueños del recurso la usan para la construcción de viviendas (palapas) y postes para cercas.

2. Exothea diphylla (Stand1.) Lundell. Sapindaceae

Nombres comunes. Wayamcox, culiché y asculiché (Península de Yucatán) (Standley y Steyermark, 1949). Ix k'ulim che', wayum koox (Yucatán, Quintana Roo) (Sosa et al., 1985).

Distribución. Península de Yucatán (Standley y Steyermark, 1949).

\section{Características macroscópicas.}

La madera presenta albura y duramen bien delimitados; la albura mide $3 \mathrm{~cm}$, es de color castaño (10YR 8/3), el duramen mide $16 \mathrm{~cm}$ y es de color rosa (7.5 YR 8/4). El olor no es característico y el sabor es ligeramente amargo, el brillo es alto, el veteado es suave, la textura es mediana y el hilo es entrecruzado.

Los poros son visibles a simple vista; el parénquima axial, los rayos, las fibras y las zonas de crecimiento con lupa.

Características microscópicas. (Lám. 1. figs. 2. a-c y cuadro 1).

La madera presenta porosidad difusa, la mayoría de los poros son solitarios y múltiples radiales de 2 a 7 , numerosos $23(18-28) / \mathrm{mm} 2$, de diámetro tangencial moderadamente pequeño 68 (45-95) $\mu \mathrm{m}$. Los elementos de vaso son de longitud mediana 408 (240-610) $\mu \mathrm{m}$, sus paredes presentan puntuaciones areoladas alternas y su placa perforada es simple. Presentan gomas.

El parénquima axial es unilateral. No presenta contenidos.

Los rayos son de 1 a 3 series, la mayoría de 2, numerosos 10 (7-13)/mm, de tipo heterogéneo III; el cuerpo está formado por células procumbentes y los extremos por células cuadradas. Los uniseriados son extremadamente bajos 108 (40-200) $\mu \mathrm{m}$, de 3 células de altura (1-4). Los poliseriados son extremadamente bajos $225(100-330) \mu \mathrm{m}$ y de anchura muy fina 23 (17-32) $\mu \mathrm{m}$. Presentan gomas.

Las fibras son de tipo libriforme y fibrotraqueidas; las libriformes son septadas, moderadamente cortas $760(460-960) \mu \mathrm{m}$, de diámetro fino $15(10-22) \mu \mathrm{m}$ y paredes delgadas 4 (2-5) $\mu \mathrm{m}$. No presentan contenidos.

\section{Usos locales.}

Los dueños del recurso la usan para la construcción de viviendas (palapas).

3. Myrcianthes fragrans (Swartz) McVaugh var. fragrans Myrtaceae

Nombres comunes. Guayabillo (Quintana Roo) (McVaugh, 1963). Xokoka'an (Yucatán y Quintana Roo) (Sosa et al., 1985). 
Distribución. Tamaulipas, Morelos, Veracruz, Chiapas, Campeche, Yucatán y Quintana Roo (McVaugh, 1963).

Características macroscópicas.

La madera presenta albura y duramen bien delimitados; la albura mide $3 \mathrm{~cm}$, es de color rosa (5YR 8/3) y rosa grisáceo (5YR 7/2), el duramen mide $12 \mathrm{~cm}$ y es de color castaño rojizo (5YR 5/3) y castaño rojizo oscuro (5YR 2.5/1). El olor y el sabor no son característicos, el brillo es mediano, el veteado es pronunciado, la textura es fina y el hilo es recto.

Los poros, el parénquima axial, los rayos y las fibras son visibles con lupa; las zonas de crecimiento a simple vista.

Características microscópicas. (Lám. 1. figs. 3. a-c y cuadro 1).

La madera presenta porosidad difusa, los poros son solitarios, muy numerosos 52 (35-68)/mm2, de diámetro tangencial muy pequeño 33 (18-55) $\mu \mathrm{m}$. Los elementos de vaso son de longitud mediana $424(180-700) \mu \mathrm{m}$, sus paredes presentan puntuaciones areoladas alternas y su placa perforada es simple. No presentan contenidos.

El parénquima axial es unilateral, reticulado y marginal de una célula. No presenta contenidos.

Los rayos son de 1 a 3 series, la mayoría de 2, numerosos 9 (6-12)/mm, de tipo heterogéneo II y III; el cuerpo está formado por células procumbentes y los extremos por células verticales. Los uniseriados son extremadamente bajos 135 (70-300) $\mu \mathrm{m}$, de 2 células de altura (1-6). Los poliseriados son extremadamente bajos $242(100-450) \mu \mathrm{m}$ y de anchura muy fina 23 (13-33) $\mu \mathrm{m}$. Presentan gomas.

Las fibras son de tipo libriforme, moderadamente largas 1065 (750-1350) $\mu \mathrm{m}$, de diámetro fino 20 (15-25) $\mu \mathrm{m}$ y paredes muy gruesas 8 (3-10) $\mu \mathrm{m}$. Presentan sustancias amarillas.

\section{Usos locales.}

Los dueños del recurso la usan principalmente para la elaboración de muebles aunque también para palapas y durmientes.

\section{Diospyros nicaraguensis Standl. Ebenaceae}

Nombres comunes. Uchiché (Quintana Roo) (Standley y Steyermark, 1967).

Distribución. Yucatán y Quintana Roo (Standley y Steyermark, 1967).

Características macroscópicas.

La madera no presenta albura y duramen delimitados; mide $19 \mathrm{~cm}$ y es de color amarillo pálido (2.5Y 8/4), con tonalidades castaño amarillo pálido (2.5Y 6/4). El olor y el sabor no son característicos, el brillo es alto, el veteado es suave, la textura es mediana y el hilo es entrecruzado.

Los poros son visibles a simple vista; el parénquima axial, los rayos y las fibras con lupa; las zonas de crecimiento no se distinguen. 
Características microscópicas. (Lám. 1. figs. 4. a-c y cuadro 1).

La madera presenta porosidad difusa, la mayoría de los poros son solitarios, múltiples radiales de 2 a 8 y agrupados de 3 a 6 , moderadamente numerosos 12 (9-16)/mm2, de diámetro tangencial moderadamente pequeño 81 (55-112) $\mu \mathrm{m}$. Los elementos de vaso son de longitud mediana $461(230-660) \mu \mathrm{m}$, sus paredes presentan puntuaciones areoladas alternas y su placa perforada es simple. Presentan gomas.

El parénquima axial es en bandas de una sóla hilera de células y vasicéntrico. Presenta cristales romboidales.

Los rayos son de 1 a 3 series, la mayoría de 2, muy numerosos $13(11-17) / \mathrm{mm}$, de tipo heterogéneo I y III; el cuerpo está formado por células procumbentes y los extremos por células cuadradas. Los uniseriados son extremadamente bajos $348(140-870) \mu \mathrm{m}$, de 6 células de altura (1-19). Los poliseriados son muy bajos $562(260-960) \mu \mathrm{m}$ y de anchura moderadamente fina $31(20-60) \mu \mathrm{m}$. Presentan gomas y cristales romboidales.

Las fibras son de tipo libriforme, medianas $999(600-1300) \mu \mathrm{m}$, de diámetro fino 17 (12-25) $\mu \mathrm{m}$ y paredes delgadas 5 (2-10) $\mu \mathrm{m}$. No presentan contenidos.

\section{Usos locales.}

Los dueños del recurso la usan para la construcción de viviendas (palapas).

5. Thevetia gaumeri Hemsl. Apocynaceae

Nombres comunes. Ajkits, aki'its, k'aan lool, sakits, akits de playa; campanilla, cojón de gato, cojón de toro (Campeche, Yucatán y Quintana Roo) (Sosa et al., 1985).

Distribución. Campeche, Yucatán y Quintana Roo (Standley y Williams, 1969).

Características macroscópicas.

La madera presenta albura y duramen bien delimitados; la albura mide $4 \mathrm{~cm}$, es de color castaño muy pálido (10YR $8 / 3$ y 8/4), el duramen mide $20 \mathrm{~cm}$ y tiene varias tonalidades: castaño (10YR 5/3), castaño oscuro (10YR 3/3) y castaño amarillento oscuro (10YR 3/4). El olor es a aceite y el sabor no es característico, el brillo es mediano, el veteado es pronunciado, la textura es fina y el hilo es recto.

Los poros, el parénquima axial, los rayos y las fibras son visibles con lupa; las zonas de crecimiento a simple vista.

Características microscópicas. (Lám. 1. figs. 5. a-c y cuadro 1).

La madera presenta porosidad difusa, la mayoría de los poros son múltiples radiales de 2 a 7 , solitarios y agrupados de 2 a 9 , muy numerosos $40(26-56) / \mathrm{mm} 2$, de diámetro tangencial moderadamente pequeño $52(25-70) \mu \mathrm{m}$. Los elementos de vaso son de longitud mediana $522(250-880) \mu \mathrm{m}$, sus paredes presentan puntuaciones areoladas alternas y su placa perforada es simple. No presentan contenidos.

El parénquima axial es difuso, unilateral y marginal. Presenta abundantes cristales romboidales.

Los rayos son de 1 a 4 series, la mayoría de 1 , numerosos $10(6-14) / \mathrm{mm}$, de tipo heterogéneo II y III; el cuerpo está formado por células procumbentes y los extremos por células verticales y cuadradas. Los uniseriados son extremadamente bajos 275 
(120-600) $\mu \mathrm{m}$, de 6 células de altura (1-17). Los poliseriados son extremadamente bajos $387(160-800) \mu \mathrm{m}$ y de anchura moderadamente fina $33(20-50) \mu \mathrm{m}$. No presentan contenidos.

Las fibras son de tipo libriforme, moderadamente largas 1085 (750-1500) $\mu \mathrm{m}$, de diámetro fino 22 (15-32) $\mu \mathrm{m}$ y paredes delgadas 5 (3-10) $\mu \mathrm{m}$. No presentan contenidos.

Usos locales.

Los dueños del recurso la usan para la construcción de viviendas (palapas) y en la elaboración de durmientes.

AGRADECIMIENTOS. Las autoras agradecen al Centro de Investigaciones de Quintana Roo (CIQRO), en especial al Biól. Odilón Sánchez y al Sr. Honorato Huitzil las facilidades brindadas en la recolección de las especies; al Dr. Hermilo Quero de la Universidad Nacional Autónoma de México, a la M. en C. Rosaura Grether y al Dr. Ramón Riba de la Universidad Autónoma Metropolitana-Iztapalapa, la revisión del manuscrito. El presente trabajo tuvo financiamiento parcial de la SEP-DIGICSA (Convenio 085-01-0271).

\section{LITERATURA CITADA}

CÁRDENAS, E. 1971. Estudio anatómico de la madera de ocho especies de leguminosas. Tesis. Escuela Nacional de Ciencias Biológicas. IPN. México. 54p.

CHATtAWAY, M. 1932. Proposed standards for numerical values used in describing woods. Trop. Woods 29:20-28.

CORRAL, G. 1985. Características anatómicas de la madera de once especies tropicales. Bol. Téc. Inst. Nac. Invest. For. México. No. 127:66.

DALLA TORRE, C. DEy H. HARMS. 1963. Genera siphonogamarum ad systema Engle rianum conscripta. Reprint: Wiesbaden. Verlag für Wissenschffliche Neudrucke GMBH. 637p.

ESCALANTE, S. 1986. La flora del Jardín Botánico del Centro de Investigaciones de Quintana Roo, A. C. Tesis. Facultad de Ciencias Biológicas. Universidad Veracruzana. Xalapa, Ver. 163p.

FLORES, R. L. 1981. Anatomía de la madera de tres especies tropicales mexicanas. Bol. Téc. Inst. Nac. Invest. For. México. No. 24:13.

GURIDI, L. 1968. Anatomía de la madera de cinco especies tropicales de importancia económica. Tesis. Facultad de Ciencias. UNAM. México. 34p.

HALFFTER, G. 1980. Colonización y conservación de recursos bióticos en el trópico. INIREB. Xalapa, Ver. $47 \mathrm{p}$.

HuERTA, J. y J. BECERRA. 1982. Anatomía macroscópica y algunas características físicas de diecisiete maderas tropicales mexicanas. Bol. Div. Inst. Nac. Invest. For. México. No. 46: 61.

IAWA COMMITTEE. 1937. Standard terms of length of vessel members and wood fibers. Trop. Woods 51:21.

IAWA COMMITTEE. 1939. Standard terms of size for vessel diameter and ray width. Trop. Woods 59:51-52. 


\section{ANATOMIA DE LA MADERA DE QUINTANA ROO}

IAWA COMMITTEE. 1989. IAWA list of microscopic features for hardwood identification. IAWA Bull. n.s. $10(3): 219-332$.

JOHANSEN, D. 1940. Plant microtechnique. McGraw-Hill, New York. 523p.

KRIBS, D. 1968. Commercial foreing woods on the american market. Dover Publications Inc., New York. 241p.

KUKACHKA, B. 1977. Sectioning refractory woods for anatomical studies. USDA. Forest Service Research Note FPL-0236:1-9.

McVAUGH, R. 1963. Tropical American Myrtaceae. II. Fieldiana Bot. 29(8):485-489.

MiRANDA, F. 1978. Vegetación de la Península Yucateca. En: Los recursos naturales del sureste y su aprovechamiento. Tomo II. IMERNAR, A. C. México, D. F. pp. 161-271.

MUNSELL COLOR COMPANY. 1954. Munsell soil color charts. Baltimore, Maryland 17p.

Paz-PÉrez Olvera, C. DE LA, F. Robles y A. Simental. 1979. Determinación de las características anatómicas y físico-mecánicas de la madera de 4 especies de leguminosas. Bol. Téc. Inst. Nac. Invest. For. México. No. 61:35.

Paz-PÉrez Olvera, C. DE LA, T. CArmona y A. Rogel. 1980. Estudio anatómico de la madera de 43 especies tropicales. Bol. Téc. Inst. Nac. Invest. For. México. No. 63:272.

RAMOS, C. y V. DÍAZ. 1981. Instrucciones para colectar muestras de maderas para estudios tecnológicos. Bol. Div. Inst. Nac. Invest. For. México. No. 54:15.

RebOllar, S., C. De LA PAZ-PÉREZ OlverA y A. QUiNTANAR. 1987. Maderas de la Península de Yucatán. I. Estudio anatómico de la madera de 3 especies del estado de Yucatán. Biótica 12(3):159-179.

RoGEL, A. 1982. Características anatómicas de la madera de siete especies tropicales. Bol. Téc. Inst. Nac. Invest. For. México. No. 86:54.

SÁNCHEZ, O. 1987. Estructura y composición de la selva mediana subperennifolia presente en el Jardín Botánico del CIQRO, Puerto Morelos, Quintana Roo. Tesis. Facultad de Biología, Universidad Veracruzana. Xalapa, Ver. 61p.

SCHULTZ, H. y N. von GROTTHUS. 1968. Investigación de algunas especies arbóreas de los bosques tropicales de México. I. México y Sus Bosques. Epoca III (23):9-17.

SCHULTZ, H. y N. von GROTTHUS. 1968a. Investigación de algunas especies arbóreas de los bosques tropicales de México. II. México y Sus Bosques. Epoca III (24):18-25.

SCHULTZ, H. y N. von GROTTHUS. 1969. Investigación de algunas especies arbóreas de los bosques tropicales de México. III. México y Sus Bosques. Epoca III. (25):4-22.

SOSA, V., S. Flores, V. RicO-GRAY, R. LiRA y J. ORTíZ. 1985. Lista florística y sinonimia maya. En: Etnoflora Yucatanense. Fascículo 1. INIREB, Xalapa, Ver. 225p.

StANDley, P. y J. STEYERMARK. 1949. Euphorbiaceae. En: Flora of Guatemala. Fieldiana Bot. 24(6):89-90.

STANDLEY, P.y J.STEYERMARK. 1949. Sapindaceae. En: Flora of Guatemala. Fieldiana Bot. 24(6):247248.

STANDLEY, P.y J. STEYERMARK. 1967. Ebenaceae. En: Flora of Guatemala. Fieldiana Bot. 24(8):250.

STANDLEY, P.y L. WiLliAMS. 1969. Apocynaceae. En: Flora of Guatemala. Fieldiana Bot. 24(8):396.

TORRES, R. 1969. Descripción macroscópica comparativa de 25 especies de maderas tropicales de importancia económica (clave de identificación). Tesis. Escuela Nacional de Agricultura Chapingo. México. 109 p.

TORTORELLI, L. 1956. Maderas y bosques argentinos. ACME, Buenos Aires. 910p. 
BOLETIN DE LA SOCIEDAD BOTANICA DE MEXICO, Núm. 53, 1993

TRUEBA, J. 1983. La problemática forestal y su incidencia en el medic ambiente. En: Ecología y Recursos Naturales. Hacia una política ecológica del PSUM. Ediciones del Comité Central, México. pp. 53-64. 